\title{
Coverage of curves in 3D with swarms of nonholonomic aerial robots
}

\author{
Mateus M. Gonçalves, Luciano C. A. Pimenta, \\ Guilherme A. S. Pereira \\ School of Engineering, Universidade Federal de Minas Gerais - UFMG \\ (e-mail: \{mateusmg,lucpim,gpereira\}@cpdee.ufmg.br).
}

\begin{abstract}
This paper presents a vector field based methodology for coverage of curves in threedimensional spaces with large groups of fixed-wing aerial robots. We model the robots as spheres with an associated nonholonomic kinematic model with minimum forward speed constraint. Under certain assumptions we guarantee that the robots will converge to and circulate along a desired curve avoiding inter-robot collisions while satisfying the constraints.
\end{abstract}

Keywords: Robotics, Cooperative control, Nonholonomic systems, Autonomous vehicles.

\section{INTRODUCTION}

The main goal of this paper is to propose a solution to control a large team of fixed-wing aerial robots to converge to a curve in their three-dimensional workspace while avoiding inter-robot collision. Since these airplane based Unmanned Air Vehicles (UAVs) need to have a minimum air speed, it is also important that the robots circulate the desired curve with a positive linear velocity. Because we are thinking in teams with tenths or hundreds of robots, often called swarms, it is also mandatory to program the robots with simple, identical and decentralized controllers that rely on local sensing and limited communication.

Although convergence of teams of mobile robots to curves is not a recent problem (see, for example, the work by Sugihara and Suzuki (1996)), most of the solutions found in the literature only include two-dimensional environments and fully actuated, holonomic mobile robots. This is the case, for example, of the work by Hsieh et al. (2008), which presents provable correct and decentralized solutions to control large groups of fully actuated mobile robots to converge to closed curves. When the methodology does not implicitly consider the nonholonomic constraints of the robots, some authors apply static feedback linearization controllers to make actual robots to track a virtual holonomic robot (Chaimowicz et al., 2005). Other authors have explicitly considered the nonholonomic constraints in their solutions (Ceccarelli et al., 2008), but most of solutions are only valid to a specific model of robot, in general a unicycle, that may turn in place.

Regarding the robot workspace dimension, just a few authors have proposed methodologies that control mobile robots in three-dimensional workspaces. Lawrence et al. (2009) proposes a 3D vector field to guide a single mobile robot to converge to planar curves while Gonçalves et al. (2010) have a similar methodology, but considers generic, time-varying curves. By the authors knowledge, no methodology has been proposed thus far that controls teams of robots to converge to curves in $3 \mathrm{D}$.
The solution we propose in this paper for multirobot convergence to curves in $3 \mathrm{D}$ is based on the vector field proposed by our group in (Gonçalves et al., 2010). This is based on a sum of two terms: (i) a gradient term, that attracts the robot to the curve, and (ii) a rotational term orthogonal to the first one, that makes the robot circulate the curve. To avoid collisions among the robots we divide the controller in two steps. In the first step, the robots only follow a projection of the vector field, which is modulated so that the group can homogeneously distribute itself around the curve. In this step each robot has a unique fixed altitude and collision avoidance is not an issue. In the second step, the robots follow the complete vector field with its magnitude modulated to make the robots' angular velocity around the curve constant.

\section{PROBLEM STATEMENT}

Let the two functions $\alpha_{1}: \mathbb{R}^{3} \mapsto \mathbb{R}$ and $\alpha_{2}: \mathbb{R}^{3} \mapsto \mathbb{R}$ be defined as

$$
\begin{aligned}
& \alpha_{1}=\zeta_{1}(x, y), \\
& \alpha_{2}=z-\zeta_{2}(x, y),
\end{aligned}
$$

where $\zeta_{1}$ and $\zeta_{2}$ are functions with continuous partial derivatives, and $x, y$, and $z$ are Cartesian coordinates.

A level surface of $\alpha_{1}$ or $\alpha_{2}$ is defined as

$$
\mathcal{M}_{C}\left(\alpha_{i}\right):\left\{\mathbf{q} \in \mathbb{R}^{3} \mid \alpha_{i}(\mathbf{q})=C\right\}, i=1,2, C \in \mathbb{R} .
$$

In this paper, function $\alpha_{1}$ is defined such that, for any $C \in \mathbb{R}$ the projection of $\mathcal{M}_{C}\left(\alpha_{1}\right)$ onto the $x y$-plane, defined as $\Pi_{x y}\left(\mathcal{M}_{C}\left(\alpha_{1}\right)\right)$, is closed.

Consider now the curves $\Gamma_{C}^{D}$ resulting from the intersection of the level surfaces of $\alpha_{1}$ and $\alpha_{2}$ :

$$
\begin{aligned}
& \Gamma_{C}^{D}=\mathcal{M}_{C}\left(\alpha_{1}\right) \cap \mathcal{M}_{D}\left(\alpha_{2}\right), \\
& C \in \mathbb{R}, D \in \mathbb{R} .
\end{aligned}
$$

The target curve $\Gamma$ is defined as:

$$
\Gamma=\Gamma_{0}^{0} .
$$

Let $\Omega$ be a finite set of $N$ spherical robots, 


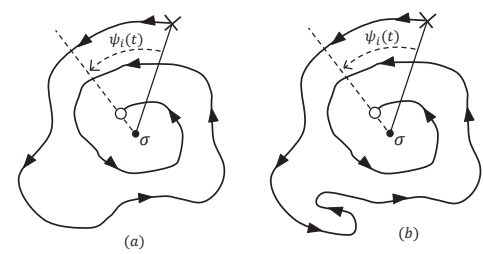

Fig. 1. (a) A single-oriented path projection $\mathcal{P}^{\prime}$. (b) A path projection that is not single-oriented.

$$
\Omega=\left\{\bigcup_{i=1}^{N} \omega_{i}\right\}
$$

such that to a given robot $\omega_{i}$ is associated a sphere radius $R_{i}$ and a sphere center in Cartesian coordinates $\mathbf{q}_{i}=\left[\begin{array}{lll}x_{i} & y_{i} & z_{i}\end{array}\right]^{T}$ measured from a global fixed reference frame with origin denoted as the point $\boldsymbol{\sigma}=\left[\begin{array}{lll}0 & 0 & 0\end{array}\right]^{T}$. We will assume that the group of robots is homogeneous which means that $R_{i}=R, \forall i$. Furthermore, for each robot we assume the kinematic model given by

$$
\dot{\mathbf{q}}_{i}=\left\{\begin{array}{l}
\dot{x}_{i}=v_{f}^{i} \cos \theta_{i} \\
\dot{y}_{i}=v_{f}^{i} \sin \theta_{i} \\
\dot{z}_{i}=v_{z}^{i}
\end{array},\right.
$$

where $\theta_{i}$ is the robot yaw angle. The evolution of this angle is governed by $\dot{\theta}_{i}=v_{\theta}^{i}$. The system control inputs are $v_{\theta}^{i}$ (yaw angle variation rate), $v_{f}^{i}$ (forward speed), and $v_{z}^{i}$ (altitude variation rate). The minimum forward speed constraint is also considered:

$$
v_{f}^{i} \geq v_{\min }>0 .
$$

Also, consider the following variables:

$$
\begin{aligned}
& \psi_{i}=\operatorname{atan} 2\left(y_{i}, x_{i}\right) \in[0,2 \pi)-\text { the azimuthal angle, } \\
& \mathbf{r}_{i}=\left[\begin{array}{c}
x_{i} \\
y_{i}
\end{array}\right] \in \mathbb{R}^{2} \text { - the radial vector } \text { and } \\
& \hat{\boldsymbol{\psi}}_{i}=\frac{1}{\left\|\mathbf{r}_{i}\right\|}\left[\begin{array}{c}
-y_{i} \\
x_{i}
\end{array}\right] \text { - the azimuth direction vector. }
\end{aligned}
$$

We now define a path in Cartesian coordinates as:

$$
\mathcal{P}_{i}=\left\{\mathbf{q}_{i}(t) \in \mathbb{R}^{3}, t \geq 0\right\}
$$

and the projection of this path:

$$
\mathcal{P}_{i}^{\prime}=\left\{\Pi_{x y}\left(\mathbf{q}_{i}(t)\right) \equiv\left[x_{i} y_{i}\right]^{T} \in \mathbb{R}^{2}, t \geq 0\right\} .
$$

The path projection $\mathcal{P}_{i}^{\prime}$ is single-oriented if

$$
\frac{d}{d t} \psi_{i}>0
$$

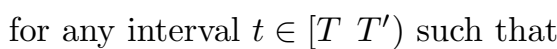

$$
\psi_{i}(T)=0, \quad \psi_{i}\left(T^{\prime}\right)=2 \pi .
$$

An example and a counter-example of a single-oriented path projection are shown in Figure 1.

Two definitions concerning the robots' paths are now given:

Definition 1. A path $\mathcal{P}_{i}$ converges to a curve $\Gamma_{C}^{D}$ if

$$
\lim _{t \rightarrow \infty} \mathbf{q}_{i}(t) \in \Gamma_{C}^{D} \text {. }
$$

Definition 2. A path $\mathcal{P}_{i}$ circulates a curve $\Gamma_{C}^{D}$ if it converges to $\Gamma_{C}^{D}$ and its path projection $\mathcal{P}_{i}^{\prime}$ is singleoriented.

Based on the definitions above, we are finally able to state the problem to be solved.

Problem Statement Drive each robot $\omega_{i} \in \Omega$ such that the robot path $\mathcal{P}_{i}$ circulates the target curve $\Gamma$ while it avoids collisions with other robots.

Collision avoidance is guaranteed when:

$$
\begin{aligned}
& \left\|\mathbf{q}_{i}(t)-\mathbf{q}_{j}(t)\right\|-2 R>0 \\
& \forall t \geq 0, \quad \forall\left(\omega_{i}, \omega_{j}\right) \in[\Omega \times \Omega], \omega_{i} \neq \omega_{j} .
\end{aligned}
$$

\section{METHODOLOGY}

This section presents a methodology that solves the stated problem. Without loss of generality we will assume circulation in the counterclockwise direction.

We start with the definitions of neighbor robots and phase homogeneization.

Definition 3. If the set $\Omega_{i}^{\prime}$ is given by $\Omega_{i}^{\prime}=\Omega-\omega_{i}$, the neighbors of the robot $\omega_{i} \in \Omega$ are

$$
\begin{aligned}
& \omega_{i}^{L}=\underset{\omega_{j} \in \Omega_{i}^{\prime}}{\operatorname{argmin}}\left\{\tilde{\psi}_{j}\right\}, \\
& \omega_{i}^{R}=\underset{\omega_{j} \in \Omega_{i}^{\prime}}{\operatorname{argmax}}\left\{\tilde{\psi}_{j}\right\},
\end{aligned}
$$

where $\tilde{\psi}_{j}$ is the measure of $\psi_{j}$ taken with respect to $\omega_{i}$, i.e. $\tilde{\psi}_{i}=0$.

Definition 4. Given a robot $\omega_{i} \in \Omega$ and its neighbors $\omega_{i}^{L}$ and $\omega_{i}^{R}$, define $\tilde{\psi}_{i}^{L}$ and $\tilde{\psi}_{i}^{R}$ their respective angles measured from $\omega_{i}$. The phase homogeneization of the set $\Omega$ is a process where every robot $\omega_{i} \in \Omega$ traverse a path $\mathcal{P}_{i}$ such that all robots become evenly distributed in the phase (azimuthal angle) space after a time $t=T$, i. e.,

$$
\begin{aligned}
& \left.\left|\tilde{\psi}_{i}^{L}(t)\right|=\mid \tilde{\psi}_{i}^{R}(t)\right)-2 \pi \mid=\frac{2 \pi}{N} \\
& \forall \omega_{i} \in \Omega, t \geq T
\end{aligned}
$$

where $N$ is the number of elements of the set $\Omega$. Furthermore, the collision between robots is avoided during the rearrangement, and after (6) is attained, all the robots move with the same azimuthal angle velocity

$$
\lim _{t \rightarrow T} \frac{d}{d t} \psi_{i}=\dot{\psi}_{f} \forall t \geq T \text {. }
$$

Our approach is divided in two steps: before and after the phase homogeneization. The end of the first step is ensured by reaching the condition (6). In both steps we use an artificial vector field to define the direction of motion.

In our previous work (Gonçalves et al., 2010), we presented a vector field which allows for convergence and circulation of a single robot along time-varying curves embedded in $n$ dimensional spaces. In the present paper, we use a similar vector field $\boldsymbol{\mu}: \mathbb{R}^{3} \mapsto \mathbb{R}^{3}$ given by:

$$
\boldsymbol{\mu}(\mathbf{q})=-\lambda(\mathbf{q}) \nabla \varphi(\mathbf{q})-\rho(\mathbf{q})\left[\nabla \times\left(\alpha_{1}(\mathbf{q}) \nabla \alpha_{2}(\mathbf{q})\right)\right],
$$

where

$$
\varphi(\mathbf{q})=A \alpha_{1}(\mathbf{q})^{2}+\alpha_{2}(\mathbf{q})^{2}, A>0, A \text { constant },
$$


and

$$
\begin{aligned}
& \lambda(\mathbf{q})=\frac{\dot{\psi}_{f}\|\mathbf{r}\|}{\left[\hat{\boldsymbol{\psi}}^{T} \frac{\Pi_{x y}\left(\boldsymbol{\mu}^{\prime}(\mathbf{q})\right)}{\left\|\Pi_{x y}\left(\boldsymbol{\mu}^{\prime}(\mathbf{q})\right)\right\|}\right]} \frac{\lambda^{\prime}(\mathbf{q})}{\left\|\boldsymbol{\mu}^{\prime}(\mathbf{q})\right\|}, \\
& \rho(\mathbf{q})=\frac{\dot{\psi}_{f}\|\mathbf{r}\|}{\left[\hat{\boldsymbol{\psi}}^{T} \frac{\Pi_{x y}\left(\boldsymbol{\mu}^{\prime}(\mathbf{q})\right)}{\left\|\Pi_{x y}\left(\boldsymbol{\mu}^{\prime}(\mathbf{q})\right)\right\|}\right]} \frac{\rho^{\prime}(\mathbf{q})}{\left\|\boldsymbol{\mu}^{\prime}(\mathbf{q})\right\|},
\end{aligned}
$$

with

$$
\left.\boldsymbol{\mu}^{\prime}(\mathbf{q})\right)=-\lambda^{\prime}(\mathbf{q}) \nabla \varphi(\mathbf{q})-\rho^{\prime}(\mathbf{q})\left[\nabla \times\left(\alpha_{1}(\mathbf{q}) \nabla \alpha_{2}(\mathbf{q})\right)\right],
$$

where $\lambda^{\prime}(\mathbf{q}), \rho^{\prime}(\mathbf{q})$ are strictly positive and continuous functions. Considering the notation $\boldsymbol{\mu}=\left[\begin{array}{ll}\mu_{x} & \mu_{y} \\ \mu_{z}\end{array}\right]^{T}$, the proposed control law for a robot with kinematic model as in (3) is given by

$$
v_{\theta}^{i}=\dot{\theta}_{i}^{d}-K_{\theta}\left(\theta_{i}-\theta_{i}^{d}\right),
$$

where $\theta_{i}^{d}=\operatorname{atan} 2\left(\mu_{y}, \mu_{x}\right), \dot{\theta}_{i}^{d}=\frac{d}{d t}\left[\operatorname{atan} 2\left(\mu_{y}, \mu_{x}\right)\right]$, and $K_{\theta}>0$.

$$
v_{f}^{i}=\max \left\{\frac{\left(\frac{d}{d t} \psi_{i}\right)\left\|\mathbf{r}_{i}\right\|}{\left[\hat{\boldsymbol{\psi}}^{T} \frac{\Pi_{x y}\left(\dot{\mathbf{q}}_{i}\right)}{\left\|\Pi_{x y}\left(\dot{\mathbf{q}}_{i}\right)\right\|}\right]}, v_{\min }\right\},
$$

with

$$
\frac{d}{d t} \psi_{i}=\dot{\psi}_{f}+K_{\psi} \bar{\psi}_{i}
$$

where

$$
\bar{\psi}_{i}=\frac{\tilde{\psi}_{i}^{L}+\tilde{\psi}_{i}^{R}-2 \pi}{2}
$$

and $K_{\psi}>0, \dot{\psi}_{f}>0$. Finally, $v_{z}^{i}=\left\{\begin{array}{ll}0 & \text { during phase homogeneization or if } t<T_{a} \\ \mu_{z} & \text { otherwise }\end{array}\right.$,

where $T_{a}$ is the time of alignment with the vector field. After this time we assume the robot yaw angle $\theta_{i}$ is equal to the angle of the field projection onto the $x y$-plane, $\theta_{i}^{d}$.

The proposed strategy is decentralized since each robot relies only on its own state information and of its two neighbors. Next section will present mathematical guarantees for the proposed strategy. Our analysis is built upon the following assumptions:

(1) The set $\mathcal{Q}:\left\{\mathbf{q} \in \mathbb{R}^{3} \mid \nabla \alpha_{1}(\mathbf{q})=\mathbf{0}\right\}$ must be repulsive, i.e., every path $\mathcal{P}_{i}$ induced by $\boldsymbol{\mu}$ (that is, $\dot{\mathbf{q}}_{i}(t)=$ $\left.\boldsymbol{\mu}\left(\mathbf{q}_{i}(t)\right)\right)$ has the property

$$
\mathbf{q}_{i}(0) \notin \mathcal{Q} \Rightarrow \mathbf{q}_{i}(t) \notin \mathcal{Q} \forall t>0 .
$$

Furthermore, $\mathbf{q}_{i}(0) \notin \mathcal{Q} \forall \omega_{i} \in \Omega$;

(2) For any path $\mathcal{P}_{i}$, induced by $\boldsymbol{\mu}, \omega_{i} \in \Omega$

$$
\hat{\boldsymbol{\psi}}\left(\mathbf{q}_{i}\right)^{T} \Pi_{x y}\left(\boldsymbol{\mu}\left(\mathbf{q}_{i}\right)\right)>0 \forall t \geq 0 ;
$$

(3) The initial condition for the robots yaw angle is such that $\left|\theta_{i}-\theta_{i}^{d}\right|<\pi / 2$.

(4) We compute $\rho$ and $\lambda$ such that

$$
\frac{\rho^{\prime}\left(\mathbf{q}_{i}\right)}{\lambda^{\prime}\left(\mathbf{q}_{i}\right)}>\left|\frac{\left\|\mathbf{r}_{i}\right\| \hat{\boldsymbol{\psi}}\left(\mathbf{q}_{i}\right)^{T} \Pi_{x y}\left(\nabla \varphi\left(\mathbf{q}_{i}\right)\right)}{x_{i} \partial_{x} \alpha_{1}\left(\mathbf{q}_{i}\right)+y_{i} \partial_{y} \alpha_{1}\left(\mathbf{q}_{i}\right)}\right| \forall t>0 ;
$$

(5) The initial condition related to the relative altitude of the robots is given by

$$
\left\|\Pi_{z}\left(\mathbf{q}_{i}(t=0)\right)-\Pi_{z}\left(\mathbf{q}_{j}(t=0)\right)\right\|>2 R,
$$

\section{ANALYSIS}

Firstly, we will present three lemmas concerning the vector field given by (7). The first states that the flow induces single-oriented paths. The second states that the field will never attain a null value outside a certain set. Finally, the third shows constraints over the functions $\alpha_{1}$ and $\alpha_{2}$ that are equivalent to Assumption 2.

Lemma 1. Every path projection $\mathcal{P}_{i}^{\prime}$ traversed by a robot $\omega_{i} \in \Omega$ and induced by $\boldsymbol{\mu}$ that does not pass through the line

is single-oriented.

$$
\mathcal{L}=\left\{\begin{array}{lll}
0 & 0 & z
\end{array}\right\}^{T}, z \in \mathbb{R}
$$

Proof. In order to have a single-oriented path $\mathcal{P}_{i}$, one must have

$$
\begin{aligned}
\frac{d}{d t} \psi_{i} & =\left(\frac{1}{1+\left(\frac{y_{i}}{x_{i}}\right)^{2}}\right)\left(\frac{\dot{y}_{i} x_{i}-\dot{x}_{i} y_{i}}{\left(x_{i}\right)^{2}}\right) \\
& =\left(\frac{\dot{y_{i} x_{i}-\dot{x}_{i} y_{i}}}{\left(x_{i}\right)^{2}+\left(y_{i}\right)^{2}}\right)>0
\end{aligned}
$$

which is always finite as $\mathcal{P}_{i}$ avoids $\mathcal{L}$ and thus $x_{i} \neq 0$, $y_{i} \neq 0$. As the path is induced by $\boldsymbol{\mu}$, we have that $\dot{\mathbf{q}}_{i}=\boldsymbol{\mu}\left(\mathbf{q}_{i}\right)$. Thus the numerator in (16) can be written as

$$
\left\|\mathbf{r}_{i}\right\| \hat{\boldsymbol{\psi}}\left(\mathbf{q}_{i}\right)^{T} \Pi_{x y}\left(\boldsymbol{\mu}\left(\mathbf{q}_{i}\right)\right) .
$$

As $\left(x_{i}\right)^{2}+\left(y_{i}\right)^{2}$ is always positive, then we must have

$$
\hat{\boldsymbol{\psi}}\left(\mathbf{q}_{i}\right)^{T} \Pi_{x y}\left(\boldsymbol{\mu}\left(\mathbf{q}_{i}\right)\right)>0 .
$$

As this is guaranteed by Assumption 2, then the path projection $\mathcal{P}_{i}^{\prime}$ is single-oriented.

Lemma 2. If the set $\mathcal{Q}$ is defined as in Assumption 1, then

$$
\boldsymbol{\mu}(\mathbf{q}(t)) \neq \mathbf{0} \forall \mathbf{q} \notin \mathcal{Q} \text {. }
$$

Proof. We have

$$
\begin{aligned}
& \nabla \varphi(\mathbf{q})=2 A \alpha_{1} \nabla \alpha_{1}(\mathbf{q})+2 \alpha_{2} \nabla \alpha_{2}(\mathbf{q}), \\
& \nabla \times\left(\alpha_{1}(\mathbf{q}) \nabla \alpha_{2}(\mathbf{q})\right)=\nabla \alpha_{1}(\mathbf{q}) \times \nabla \alpha_{2}(\mathbf{q}),
\end{aligned}
$$

thus it can be concluded that

$$
\nabla \varphi(\mathbf{q}) \perp \nabla \times\left(\alpha_{1}(\mathbf{q}) \nabla \alpha_{2}(\mathbf{q})\right)
$$

and

$$
\boldsymbol{\mu}(\mathbf{q})=-\lambda(\mathbf{q}) \nabla \varphi(\mathbf{q})-\rho(\mathbf{q})\left[\nabla \times\left(\alpha_{1}(\mathbf{q}) \nabla \alpha_{2}(\mathbf{q})\right)\right] \neq 0
$$$$
\text { if } \nabla \times\left(\alpha_{1}(\mathbf{q}) \nabla \alpha_{2}(\mathbf{q})\right) \neq 0 \text {. }
$$

We have

$$
\begin{aligned}
\nabla \times\left(\alpha_{1}(\mathbf{q}) \nabla \alpha_{2}(\mathbf{q})\right) & =\left(\partial_{y} \alpha_{1} \partial_{z} \alpha_{2}-\partial_{z} \alpha_{1} \partial_{y} \alpha_{2}\right) \hat{\mathbf{i}} \\
& +\left(\partial_{z} \alpha_{1} \partial_{x} \alpha_{2}-\partial_{x} \alpha_{1} \partial_{z} \alpha_{2}\right) \hat{\mathbf{j}} \\
& +\left(\partial_{x} \alpha_{1} \partial_{y} \alpha_{2}-\partial_{y} \alpha_{1} \partial_{x} \alpha_{2}\right) \hat{\mathbf{k}}
\end{aligned}
$$

However

and then,

$$
\begin{aligned}
& \partial_{z} \alpha_{2}=\partial_{z}\left[z-\zeta_{2}(x, y)\right]=1 \\
& \partial_{z} \alpha_{1}=\partial_{z} \zeta_{1}(x, y)=0
\end{aligned}
$$

$$
\begin{aligned}
\nabla \times\left(\alpha_{1}(\mathbf{q}) \nabla \alpha_{2}(\mathbf{q})\right) & =\left(\partial_{y} \alpha_{1}\right) \hat{\mathbf{i}} \\
& -\left(\partial_{x} \alpha_{1}\right) \hat{\mathbf{j}} \\
& +\left(\partial_{x} \alpha_{1} \partial_{y} \alpha_{2}-\partial_{y} \alpha_{1} \partial_{x} \alpha_{2}\right) \hat{\mathbf{k}}
\end{aligned}
$$

with

$$
\left\|\nabla \times\left(\alpha_{1}(\mathbf{q}) \nabla \alpha_{2}(\mathbf{q})\right)\right\|^{2} \geq\left\|\nabla \alpha_{1}(\mathbf{q})\right\|^{2} .
$$


Lemma 3. Assumption 2 is equivalent to the following condition:

$$
x \partial_{x} \alpha_{1}(\mathbf{q})+y \partial_{y} \alpha_{1}(\mathbf{q})>0 \forall \mathbf{q} \in \mathbb{R}^{3}-\mathcal{L} .
$$

Proof. Suppose that $\lambda\left(\mathbf{q}_{i}\right)=0$ at time $t$. Thus,

$$
\begin{aligned}
& \boldsymbol{\mu}=-\rho\left(\mathbf{q}_{i}\right)\left[\nabla \times\left(\nabla \alpha_{1}\left(\mathbf{q}_{i}\right) \alpha_{2}\left(\mathbf{q}_{i}\right)\right)\right], \\
& \Pi_{x y}(\boldsymbol{\mu})=-\rho\left(\mathbf{q}_{i}\right)\left[\partial_{y} \alpha_{1}\left(\mathbf{q}_{i}\right)-\partial_{x} \alpha_{1}\left(\mathbf{q}_{i}\right)\right]^{T} .
\end{aligned}
$$

Assumption 2 is given by

However

$$
\hat{\boldsymbol{\psi}}\left(\mathbf{q}_{i}\right)^{T} \Pi_{x y}\left(\boldsymbol{\mu}\left(\mathbf{q}_{i}\right)\right)>0 .
$$

$$
\begin{aligned}
& \hat{\boldsymbol{\psi}}\left(\mathbf{q}_{i}\right)^{T} \Pi_{x y}\left(\boldsymbol{\mu}\left(\mathbf{q}_{i}\right)\right)= \\
& \quad=-\rho\left(\mathbf{q}_{i}\right)\left[-y_{i} x_{i}\right]\left[\partial_{y} \alpha_{1}\left(\mathbf{q}_{i}\right)-\partial_{x} \alpha_{1}\left(\mathbf{q}_{i}\right)\right]^{T} /\left\|\mathbf{r}_{i}\right\| \\
& \quad=\rho\left(\mathbf{q}_{i}\right)\left(x_{i} \partial_{x} \alpha_{1}\left(\mathbf{q}_{i}\right)+y_{i} \partial_{y} \alpha_{1}\left(\mathbf{q}_{i}\right)\right) /\left\|\mathbf{r}_{i}\right\| .
\end{aligned}
$$

As $\rho\left(\mathbf{q}_{i}\right)>0$ by definition, the condition follows. Now suppose that $\lambda\left(\mathbf{q}_{i}\right) \neq 0$. Then,

$$
\begin{aligned}
\Pi_{x y}(\boldsymbol{\mu}) & =-\lambda\left(\mathbf{q}_{i}\right) \Pi_{x y}\left(\nabla \varphi\left(\mathbf{q}_{i}\right)\right) \\
& -\rho\left(\mathbf{q}_{i}\right)\left[\partial_{y} \alpha_{1}\left(\mathbf{q}_{i}\right)-\partial_{x} \alpha_{1}\left(\mathbf{q}_{i}\right)\right]^{T}
\end{aligned}
$$

and, by Assumption 2,

$$
\begin{aligned}
\hat{\boldsymbol{\psi}}\left(\mathbf{q}_{i}\right)^{T} \Pi_{x y}\left(\boldsymbol{\mu}\left(\mathbf{q}_{i}\right)\right) & =-\lambda\left(\mathbf{q}_{i}\right) \hat{\boldsymbol{\psi}}\left(\mathbf{q}_{i}\right)^{T} \Pi_{x y}\left(\nabla \varphi\left(\mathbf{q}_{i}\right)\right) \\
& +\rho\left(\mathbf{q}_{i}\right)\left(x_{i} \partial_{x} \alpha_{1}\left(\mathbf{q}_{i}\right)+y_{i} \partial_{y} \alpha_{1}\left(\mathbf{q}_{i}\right)\right) /\left\|\mathbf{r}_{i}\right\|,
\end{aligned}
$$

yielding

$$
\frac{\rho\left(\mathbf{q}_{i}\right)}{\lambda\left(\mathbf{q}_{i}\right)}=\frac{\left\|\mathbf{r}_{i}\right\| \hat{\boldsymbol{\psi}}\left(\mathbf{q}_{i}\right)^{T} \Pi_{x y}\left(\nabla \varphi\left(\mathbf{q}_{i}\right)\right)}{x_{i} \partial_{x} \alpha_{1}\left(\mathbf{q}_{i}\right)+y_{i} \partial_{y} \alpha_{1}\left(\mathbf{q}_{i}\right)}>0 .
$$

A solution that is always positive can be given by

$$
\frac{\rho^{\prime}\left(\mathbf{q}_{i}\right)}{\lambda^{\prime}\left(\mathbf{q}_{i}\right)}>\left|\frac{\left\|\mathbf{r}_{i}\right\| \hat{\boldsymbol{\psi}}\left(\mathbf{q}_{i}\right)^{T} \Pi_{x y}\left(\nabla \varphi\left(\mathbf{q}_{i}\right)\right)}{x_{i} \partial_{x} \alpha_{1}\left(\mathbf{q}_{i}\right)+y_{i} \partial_{y} \alpha_{1}\left(\mathbf{q}_{i}\right)}\right| .
$$

As this is true by Assumption 4, then Assumption 2 also follows in this case. The value for the quotient $\rho\left(\mathbf{q}_{i}\right) / \lambda\left(\mathbf{q}_{i}\right)$ is always feasible (i.e., finite), as the denominator $x_{i} \partial_{x} \alpha_{1}\left(\mathbf{q}_{i}\right)+y_{i} \partial_{y} \alpha_{1}\left(\mathbf{q}_{i}\right)>0$ outside the point $x=0, y=0$ by hypothesis in this lemma.

Now a theorem proves the alignment of the robots' velocities with the vector field $\boldsymbol{\mu}$.

Theorem 1. Consider a robot $\omega_{i} \in \Omega$ with model given by (3), traversing a path $\mathcal{P}_{i}$ and controlled by the equations (9), (10), and (13). If the assumptions made in the previous section are satisfied and $t<T_{a}$, then $\theta_{i}$ converges exponentially to $\theta_{i}^{d}$ without inter-robots collisions.

Proof. Consider Equation (9), that is the controller for the yaw angle $\theta_{i}$. If we define $\epsilon=\left(\theta_{i}-\theta_{i}^{d}\right)$, then (9) can be transformed into

$$
\dot{\epsilon}=-K_{\theta} \epsilon,
$$

with solution given by

$$
\epsilon(t)=\epsilon(0) e^{-K_{\theta} t} .
$$

Thus, $\theta_{i} \rightarrow \theta_{i}^{d}$ exponentially. The absence of collisions can be verified by considering Assumption 5 and Equation (13). If $t<T_{a}$ it is guaranteed that the robots are flying at different altitudes such that their relative distance are always greater than $2 R$.

Based on Equation (19), for practical purposes we assume the time of alignment given by $T_{a}=5 \tau$, where $\tau=1 / K_{\theta}$ is the time constant.
After $T_{a}$ and homogeneization is complete we consider

$$
\Pi_{x y}\left(\dot{q}_{i}\right)=\Pi_{x y}\left(\boldsymbol{\mu}\left(\mathbf{q}_{i}(t)\right)\right)
$$

which implies, by Equation (13), that

$$
\dot{\mathbf{q}}_{i}=\boldsymbol{\mu}\left(\mathbf{q}_{i}(t)\right) .
$$

To conclude, the method for calculating the term $\dot{\theta}_{i}^{d}$ will be shown. We have

$$
\begin{aligned}
\dot{\theta}_{i}^{d}=\frac{d}{d t}\left[\operatorname{atan} 2\left(\mu_{y}, \mu_{x}\right)\right] & =\left(\frac{1}{1+\left(\frac{\mu_{y}}{\mu_{x}}\right)^{2}}\right)\left(\frac{\dot{\mu_{y}} \mu_{x}-\dot{\mu_{x}} \mu_{y}}{\mu_{x}^{2}}\right) \\
& =\left(\frac{\dot{\mu_{y} \mu_{x}-\mu_{x} \mu_{y}}}{\mu_{x}^{2}+\mu_{y}^{2}}\right),
\end{aligned}
$$

where

$$
\left[\begin{array}{l}
\dot{\mu_{x}} \\
\dot{\mu_{y}}
\end{array}\right]=\left[\begin{array}{lll}
\partial_{x} \mu_{x} & \partial_{y} \mu_{x} & \partial_{z} \mu_{x} \\
\partial_{x} \mu_{y} & \partial_{y} \mu_{y} & \partial_{z} \mu_{y}
\end{array}\right]\left[\begin{array}{c}
\dot{x} \\
\dot{y} \\
\dot{z}
\end{array}\right] .
$$

We have $\mu_{x}^{2}+\mu_{y}^{2}=\left\|\nabla \alpha_{1}\right\|^{2} \neq 0$ and thus $\dot{\theta}_{i}^{d}$ is always finite.

The next theorem guarantees the completion of the phase homogenization under assumptions.

Theorem 2. The minimum forward speed constraint $\left(v_{f}^{i} \geq\right.$ $v_{m}$ ) is always satisfied and if $t>T_{a}$ and the radial distance $\left\|\mathbf{r}_{i}\right\|$ is lower bounded, i.e. $\left\|\mathbf{r}_{i}\right\|>c$, where $c>0$, then it is always possible to find a pair $\left(\dot{\psi}_{f}, K_{\psi}\right)$ such that

$$
v_{f}^{i}\left[\hat{\boldsymbol{\psi}}^{T} \frac{\Pi_{x y}\left(\dot{\mathbf{q}}_{i}\right)}{\left\|\Pi_{x y}\left(\dot{\mathbf{q}}_{i}\right)\right\|}\right]=\left(\frac{d}{d t} \psi_{i}\right)\left\|\mathbf{r}_{i}\right\| .
$$

Proof. By Equation (10) it is straight forward that the minimum speed constraint is always satisfied. Now, we need to show that once the robots are aligned with the field $\left(t>T_{a}\right)$ we can set our parameters such that $v_{f}^{i}$ does not saturate. In order to satisfy (22) we just need to show that

$$
v_{f}^{i}=\frac{\left(\frac{d}{d t} \psi_{i}\right)\left\|\mathbf{r}_{i}\right\|}{\left[\hat{\boldsymbol{\psi}}^{T} \frac{\Pi_{x y}(\boldsymbol{\mu})}{\left\|\Pi_{x y}(\boldsymbol{\mu})\right\|}\right]} \geq v_{m} .
$$

By substituting $\dot{\psi}_{i}$ as in Equation (11), considering Assumption 2 , and the worst case: $\hat{\boldsymbol{\psi}}$ is aligned with $\Pi_{x y}(\boldsymbol{\mu})$, we obtain

$$
\dot{\psi}_{f}+K_{\psi}\left[\bar{\psi}_{i}\right] \geq \frac{v_{m}}{\left\|\mathbf{r}_{i}\right\|} .
$$

Thus, as $\left\|\mathbf{r}_{i}\right\|$ is lower bounded by $c$, and $\bar{\psi}_{i}$ is lower bounded by $-\pi$ we can always find feasible values for $\dot{\psi}_{f}$ and $K_{\psi}$.

It is proved in Franchi et al. (2010) that the controller in Equation (11) makes all the robots $\omega_{i} \in \Omega$ converge exponentially to a phase configuration given by (6) with all the robots moving with the same constant phase velocity $\dot{\psi}_{f}$. According to the proof of Theorem 2 we can choose appropriate values for $\left(\dot{\psi}_{f}, K_{\psi}\right)$ such that after $T_{a}$ the system is always able to execute the commands given by (11). Again, since we have exponential convergence for practical purposes we can assume that the phase homogeneization step ends when $t=T$ with $T$ sufficiently large.

The importance of the phase homogeneization is yet to be explained in the next section, where it will be related to the collision avoidance context. 
Now, it will be proved that each path $\mathcal{P}_{i}$ circulates the target curve $\Gamma$.

Theorem 3. A path $\mathcal{P}_{i}$ traversed by robot $\omega_{i} \in \Omega$ induced by the flow $\boldsymbol{\mu}$ converges asymptotically to $\Gamma$.

Proof. As $\mathcal{Q}$ is repulsive and $\mathbf{q}_{i}(t=0) \notin \mathcal{Q}$ by Assumption $1, \nabla \alpha_{1}\left(\mathbf{q}_{i}(t)\right) \neq \mathbf{0} \forall t>0$. Define a Lyapunov function given by

$$
\Xi\left(\alpha_{1}, \alpha_{2}\right)=\varphi\left(\mathbf{q}_{i}\right)=A \cdot \alpha_{1}\left(\mathbf{q}_{i}\right)^{2}+\alpha_{2}\left(\mathbf{q}_{i}\right)^{2}, A>0 .
$$

This function is positive-definite with

$$
\Xi(0,0) \equiv \Xi(\Gamma) \equiv 0 \text {. }
$$

If $\mathcal{P}_{i}$ is induced by $\boldsymbol{\mu}$ as the velocity field, then we have

$$
\begin{aligned}
\frac{d}{d t} \Xi\left(\alpha_{1}, \alpha_{2}\right)= & \nabla \Xi^{T} \dot{\mathbf{q}}_{i} \\
= & \nabla \varphi\left(\mathbf{q}_{i}\right)^{T} \boldsymbol{\mu}\left(\mathbf{q}_{i}\right) \\
= & -\nabla \varphi\left(\mathbf{q}_{i}\right)^{T}\left\{\lambda\left(\mathbf{q}_{i}\right) \nabla \varphi\left(\mathbf{q}_{i}\right)\right. \\
& \left.+\rho\left(\mathbf{q}_{i}\right)\left[\nabla \times\left(\alpha_{1}\left(\mathbf{q}_{i}\right) \nabla \alpha_{2}\left(\mathbf{q}_{i}\right)\right)\right]\right\} .
\end{aligned}
$$

By Equation (17)

and

$$
\nabla \varphi\left(\mathbf{q}_{i}\right) \perp \nabla \times\left(\alpha_{1}\left(\mathbf{q}_{i}\right) \nabla \alpha_{2}\left(\mathbf{q}_{i}\right)\right) \forall t \geq 0,
$$

$$
\frac{d}{d t} \Xi\left(\alpha_{1}, \alpha_{2}\right)=-\lambda\left(\mathbf{q}_{i}\right)\left\|\nabla \varphi\left(\mathbf{q}_{i}\right)\right\|^{2}
$$

Again, if the term $\nabla \varphi\left(\mathbf{q}_{i}\right)$ is expanded,

$$
\nabla \varphi(\mathbf{q})=2 A \alpha_{1} \nabla \alpha_{1}\left(\mathbf{q}_{i}\right)+2 \alpha_{2} \nabla \alpha_{2}\left(\mathbf{q}_{i}\right) .
$$

It can be noted that $\nabla \alpha_{1}\left(\mathbf{q}_{i}\right)$ and $\nabla \alpha_{2}\left(\mathbf{q}_{i}\right)$ are linearly independent and $\nabla \alpha_{2}\left(\mathbf{q}_{i}\right)$ is always non-null, as

$$
\begin{aligned}
& \partial_{z} \alpha_{2}=\partial_{z}\left[z-\zeta_{2}(x, y)\right]=1, \\
& \partial_{z} \alpha_{1}=\partial_{z} \zeta_{1}(x, y)=0 .
\end{aligned}
$$

Thus, as $\nabla \alpha_{1}\left(\mathbf{q}_{i}\right)$ is also non-null by hypothesis, it can be concluded that $2 \alpha_{1} \nabla \alpha_{1}\left(\mathbf{q}_{i}\right)+2 \alpha_{2} \nabla \alpha_{2}\left(\mathbf{q}_{i}\right)=\mathbf{0}$ if and only if $\alpha_{1}\left(\mathbf{q}_{i}\right)=0$ and $\alpha_{2}\left(\mathbf{q}_{i}\right)=0$. Then,

$$
\frac{d}{d t} \Xi\left(\alpha_{1}, \alpha_{2}\right)=-\lambda\left(\mathbf{q}_{i}\right)\left\|\nabla \varphi\left(\mathbf{q}_{i}\right)\right\|^{2}<0 \text { if } \mathbf{q}_{i} \notin \Gamma,
$$

that is, the time derivative of the Lyapunov function is negative definite. From this result, and from the fact that $\lambda\left(\mathbf{q}_{i}\right)$ and $\rho\left(\mathbf{q}_{i}\right)$ are continuous it can be concluded that the flow is stable and the induced path will asymptotically converge to $\Gamma$.

Since we proved that paths induced by the flow $\boldsymbol{\mu}$ are convergent to $\Gamma$ and we have by Lemma 1 that every path projection $\mathcal{P}_{i}^{\prime}$ that does not pass through the line $\mathcal{L}$ are single-oriented, circulation come as a corollary:

Corollary 1. A path $\mathcal{P}_{i}$ induced by the flow $\boldsymbol{\mu}$ that does not pass through $\mathcal{L}$ circulates the curve $\Gamma$.

\section{COLLISION AVOIDANCE SCHEME}

Let $\mathcal{C}$ be the smallest circle such that it is possible to verify the following property:

$$
\begin{aligned}
& \left\|\Pi_{x y}\left(\mathbf{q}_{i}\right)-\Pi_{x y}\left(\mathbf{q}_{j}\right)\right\|-2 R>0 \\
& \forall\left(\omega_{i}, \omega_{j}\right) \in[\Omega \times \Omega], \omega_{i} \neq \omega_{j},
\end{aligned}
$$

where $\Pi_{x y}\left(\mathbf{q}_{i}\right), \Pi_{x y}\left(\mathbf{q}_{j}\right) \in \mathcal{C}$ i.e., it is possible to place the circles which corresponds to the robots sphere projections onto the $x y$-plane at $\mathcal{C}$ without superposition. It must be noted that it is allowed for the robots to move in a direction outside $\mathcal{C}$, but not in a direction that points inside as collisions can possibly occur. We will consider the case where $\dot{\mathbf{q}}_{i}=\boldsymbol{\mu}(\mathbf{q})$ since after $T_{0}=\max \left\{T_{a}, T\right\}$, i.e., after alignment and phase homogeneization, we will assume that this is true. Then, the flow $\boldsymbol{\mu}$ must be adjusted in order to prevent the wrong direction of motion. This can be done as follows.

We will consider that the circle $\mathcal{C}$ is inscribed in a projected level curve $\alpha_{1}=D$, with $D<0$. If one assume also $D>0$ it may be impossible to guarantee collision avoidance and curve circulation simultaneously. It should be clear that the size of $\mathcal{C}$ naturally defines a maximum allowed number of robots $N_{\max }$. In order to avoid the projections onto the plane $x y$ of every robot $\omega_{i}$ to move into $\mathcal{C}$, we impose:

- The path projections onto the $x y$-plane $\mathcal{P}_{i}$ for every robot $\omega_{i} \in \Omega$ are outside the region $\alpha_{1}<D$ at time $t=T_{0}$.

- If

$$
\frac{d}{d t} \alpha_{1}(\mathbf{q}(t))>0, \forall \mathbf{q}(t) \in \mathcal{M}_{D}\left(\alpha_{1}\right)
$$

any path projection onto the plane $x y$ that starts with $\alpha_{1}>D$ will remain in this region for $t \geq 0$. Consequently, the robots will never reach $\mathcal{C}$ as this circle is contained in $\alpha_{1} \leq D$. We have

$$
\begin{aligned}
\frac{d}{d t} \alpha_{1}(\mathbf{q}) & =\nabla \alpha_{1}(\mathbf{q})^{T} \dot{\mathbf{q}} \\
& =\nabla \alpha_{1}(\mathbf{q})^{T} \boldsymbol{\mu}(\mathbf{q}) \\
& =-\nabla \alpha_{1}(\mathbf{q})^{T}\{\lambda(\mathbf{q}) \nabla \varphi(\mathbf{q}) \\
& \left.+\rho(\mathbf{q})\left[\nabla \times\left(\alpha_{1}(\mathbf{q}) \nabla \alpha_{2}(\mathbf{q})\right)\right]\right\} \\
& =-\lambda(\mathbf{q})\left[2 A \cdot \alpha_{1}(\mathbf{q})\left\|\nabla \alpha_{1}(\mathbf{q})\right\|^{2}+\right. \\
& \left.2 \alpha_{2}(\mathbf{q}) \nabla \alpha_{1}(\mathbf{q})^{T} \nabla \alpha_{2}(\mathbf{q})\right] .
\end{aligned}
$$

Then it is desired that

$$
\begin{aligned}
-\lambda(\mathbf{q}) & \left(A \cdot \alpha_{1}(\mathbf{q})\left\|\nabla \alpha_{1}(\mathbf{q})\right\|^{2}\right. \\
& \left.+\alpha_{2}(\mathbf{q}) \nabla \alpha_{1}(\mathbf{q})^{T} \nabla \alpha_{2}(\mathbf{q})\right)>0, \\
& \mathbf{q}(t) \in \mathcal{M}_{D}\left(\alpha_{1}\right)
\end{aligned}
$$

As $\alpha_{1}(\mathbf{q})=D<0$ then a sufficient condition is

$$
A>\frac{\left|\alpha_{2}(\mathbf{q}) \nabla \alpha_{1}(\mathbf{q})^{T} \nabla \alpha_{2}(\mathbf{q})\right|}{|D|\left\|\nabla \alpha_{1}(\mathbf{q})\right\|^{2}}, \forall \mathbf{q}(t) \in \mathcal{M}_{D}\left(\alpha_{1}\right) \text {. }
$$

A positive, non-null, constant solution (as required for $A$ ) can be given by

$$
A=\left|\frac{\max _{\mathbf{q}(t) \in \mathcal{M}_{D}\left(\alpha_{1}\right)}\left[\alpha_{2}(\mathbf{q}) \nabla \alpha_{1}(\mathbf{q})^{T} \nabla \alpha_{2}(\mathbf{q})\right]}{D \min _{\mathbf{q}(t) \in \mathcal{M}_{D}\left(\alpha_{1}\right)}\left\|\nabla \alpha_{1}(\mathbf{q})\right\|^{2}}\right|+\delta, \delta>0
$$

and this solution is always finite, as $\nabla \alpha_{1}(\mathbf{q}(t)) \neq \mathbf{0}$ by hypothesis.

After the alignment and phase homogeneization, all the robots will have path projections onto the $x y$-plane $\mathcal{P}_{i}^{\prime}$ with the same constant phase velocity $\dot{\psi}_{f}$ and thus a robot $\omega_{i}$ will have its path projection velocity $\Pi_{x y}\left(\dot{\mathbf{q}}_{i}\right)$ at the component $\hat{\boldsymbol{\psi}}\left(\mathbf{q}_{i}\right)$ given by $\dot{\psi}_{f}\left\|\mathbf{r}\left(\mathbf{q}_{i}\right)\right\|$. Besides, $\left.\left|\tilde{\psi}_{i}^{L}(t)\right|=\mid \tilde{\psi}_{i}^{R}(t)\right)-2 \pi \mid=\frac{2 \pi}{N}$. Therefore, if the number of robots $N \leq N_{\max }$ and at time $t=T_{0}$ all the robots are 

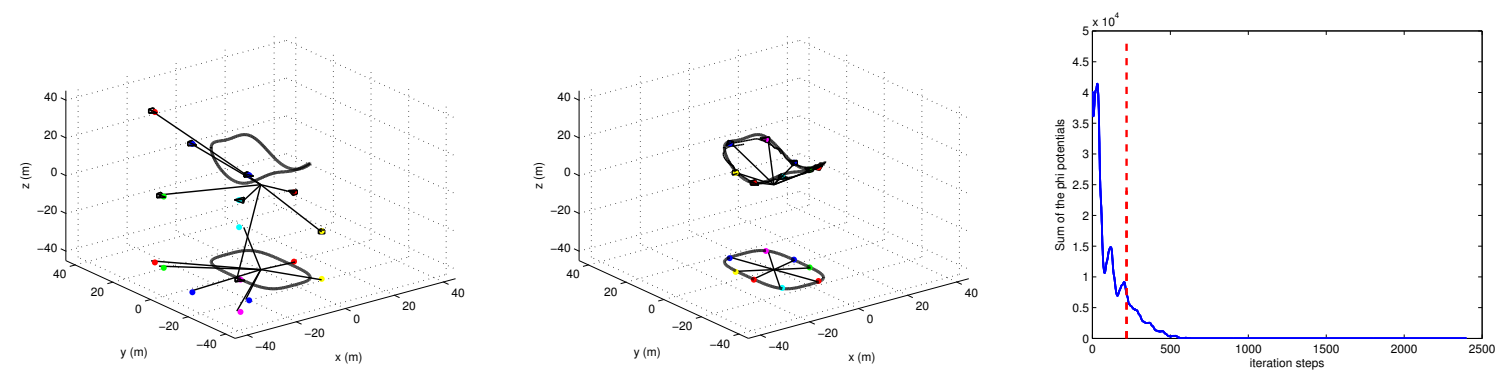

Fig. 2. A set of 8 robots with $R_{i}=2.5 m \forall \omega_{i} \in \Omega$ circulating a closed curve embedded in a $3 \mathrm{D}$ environment. The plane curve underneath is the projection of the curve and the robots onto the $x y$-plane. The graph on the right shows the evolution of the sum $\sum_{i} \varphi\left(\mathbf{q}_{i}\right)$ indicating convergence of all the robots.

in the region $\alpha_{1}>D$, collision avoidance is guaranteed if (25) is satisfied.

\section{SIMULATION RESULTS}

Now, simulation results for a set $\Omega$ of 8 robots are shown. These robots have all the same radius $R_{i}=2.5 \mathrm{~m}$, and the curves $\alpha_{1}$ and $\alpha_{2}$ are given by:

$$
\begin{aligned}
& \alpha_{1}=a x^{4}+b x^{2} y^{2}+c y^{4}-f, \\
& \alpha_{2}=z-d\left(x^{2}+y^{2}\right)+e .
\end{aligned}
$$

The values of the constants $(a, b, c, d, e$ and $f)$ are nonnull positive. The common coordinate frame origin, as required, is defined at the point $\boldsymbol{\sigma}=\left[\begin{array}{lll}0 & 0 & 0\end{array}\right]^{T}$. It must be noted that, for these functions, the condition stated in Lemma 3 that is equivalent to Assumption 2,

$$
x \partial_{x} \alpha_{1}(\mathbf{q})+y \partial_{y} \alpha_{1}(\mathbf{q})>0, \forall \mathbf{q} \in \mathbb{R}^{3}-\mathcal{L}
$$

is satisfied, as

$$
\begin{aligned}
& \partial_{x} \alpha_{1}=4 a x^{3}+2 b x y^{2} \\
& \partial_{y} \alpha_{1}=4 c y^{3}+2 b y x^{2}
\end{aligned}
$$

and

$$
x \partial_{x} \alpha_{1}+y \partial_{y} \alpha_{1}=4 a x^{4}+4 b x^{2} y^{2}+4 c y^{4}>0
$$

if $x \neq 0, y \neq 0$, that is, if $\mathbf{q} \notin \mathcal{L}$, as required. In order to have $\nabla \alpha_{1}=\mathbf{0}$, we must have:

$$
\begin{aligned}
& \partial_{x} \alpha_{1}=4 a x^{3}+2 b x y^{2}=x\left(4 a x^{2}+2 b y^{2}\right)=0 \\
& \partial_{y} \alpha_{1}=4 c y^{3}+2 b y x^{2}=y\left(4 c y^{2}+2 b x^{2}\right)=0 .
\end{aligned}
$$

The solution of the equations above is $x=0, y=$ 0 , that is, if $\mathbf{q} \in \mathcal{L}$. However as $\mathcal{C}$ defined in the last section contains the reference frame origin $\boldsymbol{\sigma}=$ $\left\{\begin{array}{lll}0 & 0 & 0\end{array}\right\}^{T}$ and has a size greater than zero, then a path $\mathcal{P}_{i}$ will never approach $\mathcal{L}$, satisfying simultaneously Assumptions 1 and 2. Assumptions 3 and 5 are ensured with proper choice of initial conditions and Assumption 4 is enforced during the online computation of the gains $\lambda\left(\mathbf{q}_{i}\right)$ and $\rho\left(\mathbf{q}_{i}\right)$. A video of the simulation can be seen at: http://coro.cpdee.ufmg.br/movies/ifac. Figure 2 shows the initial and final configuration of the circulation task. This figure also presents the evolution over time of the sum $\sum_{i} \varphi\left(\mathbf{q}_{i}\right)$. The dashed line shows the end of the homogeneization step and we can conclude that after this step the sum decays to zero indicating convergence of all the robots. In conclusion, during the simulation, convergence, circulation and collision avoidance were verified.

\section{CONCLUSIONS}

In this work we proposed a vector field based approach to guide a group of aerial robots to converge to and circulate along a static curve embedded in the $3 D$ space. We considered a simplified kinematic model which takes into account a nonholonomic constraint and a minimum forward speed constraint. The technique is decentralized since each robot can compute its control law based on its own state information and of its neighbors.

\section{ACKNOWLEDGEMENTS}

The authors gratefully acknowledge the financial support of Fundação de Amparo à Pesquisa do Estado de Minas Gerais (FAPEMIG), Conselho Nacional de Desenvolvimento Científico e Tecnológico (CNPq), and Financiadora de Estudos e Projetos (FINEP) Grant 0076/10 - Brazil. Thanks also to the IFAC Foundation for the financial support to attend the 18th IFAC World Congress under the Young Authors' Support Program.

\section{REFERENCES}

Ceccarelli, N., Marco, M.D., and Giannitrapani, A. (2008). Collective circular motion of multi-vehicle systems. Automatica, 44, 3025-3035.

Chaimowicz, L., Michael, N., and Kumar, V. (2005). Controlling swarms of robots using interpolated implicit functions. In Proc. IEEE Int. Conf. Robotics Automation, 2498-2503.

Franchi, A., Stegagno, P., Rocco, M.D., and Oriolo, G. (2010). Distributed target localization and encirclement with a multi-robot system. In 7th IFAC Symposium on Intelligent Autonomous Vehicles, 1-6.

Gonçalves, V.M., Pimenta, L.C.A., Maia, C.A., Dutra, B.C.O., and Pereira, G.A.S. (2010). Vector fields for robot navigation along time-varying curves in ndimensions. IEEE Transactions on Robotics, 26(4), 647659.

Hsieh, M.A., Kumar, V., and Chaimowicz, L. (2008). Decentralized controllers for shape generation. Robotica, 26(5), 691-701.

Lawrence, D.A., Frew, E.W., and Pisano, W.J. (2009). Lyapunov vector fields for autonomous unmanned aircraft flight control. J. Guid. Control Dyn., 31(5), 12201229 .

Sugihara, K. and Suzuki, I. (1996). Distributed algorihtms for formation of geometric patterns with many mobile robots. Journal of robotic Systems, 13(3), 127-139. 